\title{
Experimental Investigation of IEEE 802.15.4 Transmission Power Control and Interference Minimization
}

\author{
Steven Myers ${ }^{1}$, Seapahn Megerian ${ }^{1}$, Suman Banerjee ${ }^{2}$, and Miodrag Potkonjak ${ }^{3}$ \\ smmyers@wisc.edu, suman@cs.wisc.edu, megerian@ece.wisc.edu, miodrag@cs.ucla.edu \\ ${ }^{1}$ Electrical and Computer Engineering Department, University of Wisconsin Madison \\ ${ }^{2}$ Computer Science Department, University of Wisconsin Madison \\ ${ }^{3}$ Computer Science Department, University of California Los Angeles
}

\begin{abstract}
Although the characteristics of RF transmissions are physically well understood at the lowest levels of communication design, accurately incorporating power and interference effects is one of the most challenging tasks in designing efficient wireless network algorithms. The most noteworthy approaches are the circular transmission and interference "ranges" that do not apply to antennae packages that are not omnidirectional, as well as to non line-of-sight environments of many low power wireless sensor and mesh network. In this paper we experimentally investigate and then model the transmission interference of low power 802.15.4 based transceiver nodes by relying not only on distance but also on the transmit power, orientations, and the relative positions of the transmitter, receiver, and interfering nodes. The experimentally validated power/interference model is then used as the basis for an optimization algorithm that schedules nodes to minimize interference and maximize throughput from a set of senders to a set of receivers.
\end{abstract}

\section{INTRODUCTION}

Communication interference is arguably one of the most significant phenomena that can severely reduce the performance of wireless networks. Using explicitly scheduled communication links (e.g. TDMA) or through carrier sensing and collision detection techniques, interference-related performance degradations can virtually be eliminated among nodes that are within communication range, i.e. they can "hear" each other. However, in practice, two fundamental roadblocks emerge that again push the interference problem to the forefront.

First, nodes that are located too far to be able to communicate reliably can still cause significant interference problems for each other. In such cases, carrier sensing or explicit scheduling agreements between neighboring nodes may not adequately address the interference problem since the interferer is not a "neighbor".

Second, accurately modeling the actual physical interference effects in higher layer protocols is not an easy task. In addition to being very complex, accurate RF interference models require the knowledge of a large

S. Banerjee was supported in part by NSF grant mumbers CNS-0639434, CNS-0627589, CNS-0627102, and CNS-0520152 number of parameters including exact environmental characteristics, antenna and node physics, as well as noise and other stochastic phenomena.

The importance of this problem has prompted the advent of a number of simplifying models of interference to be incorporated in theoretical studies and design of protocols. Among such models, the circular communication and interference "range" model has been the most popular. This model can work well in large open spaces and for completely omnidirectional antennae. However, both the communication and the interference ranges take wildly varying shapes in practice due to non isotropic radiation patters of antenna and wireless node packages, environmental effects such as buildings and obstacles, as well as the non-uniform presence of other sources of interference and noise such as other kinds of wireless transmitters.

With the emergence of new classes of very low power wireless communication technologies for sensor networking and other long lived large scale mesh network applications, studying the interference characteristics of actual hardware nodes and developing interference minimization techniques is a fundamental research problem. With these types of networks, due to small node form factors and deployment scenarios, it is often impossible to achieve omnidirectional RF transmission (and interference) characteristics. Consequently, the relative placements and orientations of the transmitting, receiving, and interfering nodes play significant roles in the achievable communication rates.

Our strategic goal with this work is the systematic and in-depth experimental study of the interplay between interference and transmission power under a variety of conditions. Here, we focus our attention to IEEE 802.15.4 physical layer hardware that is especially well suited for low power wireless mesh networking and sensor networking applications. Although our empirical observations indicate that transmit power control is not a major contributing factor to the overall energy consumption of such low power wireless nodes, as the experimental results show in section IV, the transmit power 
level does play a significant role in terms of interference when multiple nodes are attempting to communicate in each other's proximity.

Furthermore, in this paper we investigate the interplay between interference and the relative positions and orientations of the nodes, as well as the impacts of nonsymmetric links and packet size on the achievable rates. The result of this study is the development of experimental models of interference, transmission power, and the achievable reliable communication ranges, and the subsequent use of such models in higher level algorithms such as node transmission scheduling to minimize the adverse effects of interference.

\section{A. Paper Organization}

The remainder of the paper is organized as follows: In order to position our work, we begin by briefly surveying some of the most closely related works in the next section. Section III contains the technical preliminaries and the experimental set up and hardware used to obtain the empirical results discussed and used in the rest of the paper. In section IV we present the experimental studies, results, and the corresponding analys is. In section $\mathrm{V}$ we discuss the details of our experimentally developed interference model and how it can be used in conjunction with a transmission scheduling algorithm to reduce the transmission interference and increase the throughput in multi-hop mesh networks.

\section{RELATED WORK}

Measurement-based studies of interference have received some attention in the prior literature for different wireless technologies. Most of prior measurement work in the sensor networking context has focused on the Berkeley Motes platform and its variants, which operate in the $868 / 916 \mathrm{MHz}, 433 \mathrm{MHz}$, or $315 \mathrm{MHz}$ frequency bands at nominal data rates of $19.2 \mathrm{Kbps}$. Performance measures of these systems, therefore, are expected to be different from that experienced by the 802.15.4 standard operating at the highly interfered $2.4 \mathrm{GHz}$ band with nominal data rates of $250 \mathrm{Kbps}$. We discuss a few such sensor network related measurement studies in turn.

In the domain of wireless sensor networks, some measurement studies have been conducted using the Berkeley Motes platform available from Crossbow technologies (www.xbow.com) or its variants. For example, Anatasi et. al. [1] did some experimentation and measurements on the Mica2 mote platform that utilize an RFM ChipCon radio providing a nominal bit rate of $19.2 \mathrm{Kbps}$. These motes operate in different radio frequencies including 868/916 MHz, $433 \mathrm{MHz}$ or 315 $\mathrm{MHz}$. The MAC protocol implemented on these motes is a CSMA/CA variant and is described in [2]. The goal of the authors was primarily to understand communication characteristics of motes, and in particular, effects of weather conditions on transmission ranges, and energy consumption under different operating conditions.

Zhao et. al. [4], Reijers et. al. [5], and Zhou et. al. [3] have performed other measurement studies for the same Motes platform with the focus of understanding the effects of interference. Our work in this paper is much more extensive in terms of understanding interference effects for a different sensor platform based on a 802.15.4 physical layer (from Freescale).

The work in [13] looks at the variability of radio signal strength for 802.15.4 nodes by focusing on the antenna orientation and calibration problems. Another related measurement study for the 802.15.4 standards was reported by Petrova et. al. [6] where the focus was on estimating external interference effects (e.g., due to coexistence of 802.11 based nodes) on wireless communication. Their study illustrated how packet error rates and RSSI values varied with distance between a single transmitter and a receiver. Our work, therefore, nicely complements their work by exploring self-interference effects from multiple 802.15.4 transmitters on achievable throughputs. In addition, we believe that our work provides the first detailed evaluation of various interference effects of this standard through experiments across a wide range of configurations using transmitters, receivers, and jammers, orientation of antennae, and the relative distance and elevation between nodes.

On a related note, Padhye et. al. [7] did a measurement study of interference as applicable to static multihop wireless networks, using the IEEE 802.11 standards. To use such measurements in subsequent routing decisions, the process for interference estimation needed to scale efficiently with increase in network size. The authors developed some approaches to decrease the volume the measurements needed to understand interference between any pair of wireless links in the network. Aguago et. al. [8] have performed a detailed link-level measurement study of an $802.11 \mathrm{~b}$ based wireless mesh network, and used this study to identify the performance bottlenecks in the system.

In addition to above, some recent studies such as [22] look at the effective capacity of such networks while [23] provides a mechanism for doing adaptive transmit power control.

\section{EXPERIMENTAL SETUP}

\section{A. General Hardware Description}

The hardware platform used for all the experiments in 
this paper is the Freescale ${ }^{T M}$ MC13192 Sensor Application Reference Design (SARD) device [9]. The board contains a low power 8-bit microcontroller as well as a 2.4 GHz IEEE 802.15.4 ZigBee complaint wireless transceiver. The board is powered by a 9-volt supply and includes four programmable pushbuttons, four LEDs, an in-circuit debugging module, and an RS-232 interface that is capable of baud rates up to 38400 . At the heart of the SARD board is a Motorola HCS08 family based Freescale MC9S08GT60 [9] processor, capable of running as high as $40 \mathrm{MHz}$. This processor (MCU) has a code space of $60 \mathrm{~KB}$ flash and data space of $4 \mathrm{~KB}$ RAM. Furthermore, built into the MCU is an analog to digital converter that is capable of operating in 8-bit or 10-bit modes.

Metrowerks Codewarrior ${ }^{T M} 3.0$ is used to compile the software and generate the binaries to perform the experiments. The software layers are written in both $\mathrm{C}$ and assembly with the support for the standard libraries provided by Codewarrior. Furthermore, an on-chip debugging functionality is provided though the software debugger that emulates the CPU only. The general architecture and coding paradigm is interrupt-driven.

\section{B. Wireless Transceiver}

The SARD node is equipped with an MC13192 2.4 $\mathrm{GHz}$ wireless radio [12] which is specifically designed for very low power applications such as wireless sensor networks. It requires a power supply range of 2.0 to 3.4 $\mathrm{V}$ depending on the programmable clock frequency of the MCU. The radio comes equipped with a $16 \mathrm{MHz}$ clock. It operates on one of 16 selectable channels in the $2.4 \mathrm{GHz}$ ISM band and is capable of transmit powers from $-27 \mathrm{dBm}$ to $4 \mathrm{dBm}$ with a maximum theoretical throughput of $250 \mathrm{Kbps}$. The receive sensitivity of $<-92$ $\mathrm{dBm}$ (typical) at $1.0 \%$ packet error rate, is also said to exceed that of the IEEE 802.15.4 specification of -85 $\mathrm{dBm}$. In addition to the normal operation mode, this radio also supports 3 power saving modes:

1. Off: consumes less than $1 \mu \mathrm{A}$

2. Hibernate: consumes about $1 \mu \mathrm{A}$

3. Doze: consumes about $35 \mu \mathrm{A}$

\section{Protocol Stack Implementation}

For all our experimentations, we rely on the medium access control (MAC) layer provided by Freescale for this hardware, called SMAC version 4.0 [14]. SMAC is a simple contention based protocol: When there is data available to send, it is sent immediately. There is a function provided however that measures the channel energy that would allow the implementation of CSMA/CD at a higher level. This function was not implemented for our experiments. SMAC allows for up to 123 bytes of payload data in a single packet. It also automatically appends 2 bytes of CRC to each packet in order to be able to differentiate and ignore other protocol packets and to let other protocols identify and ignore SMAC packets if needed.

Table 1. MC13192 Power Output vs. SPI Settings (from [11]). Note our experimental power settings of 4, 8, 11, and 15 .

\begin{tabular}{|c|c|c|}
\hline $\begin{array}{c}\text { PA Power Adjust } \\
\text { Reg 127:0] } \\
\text { (Hex) }\end{array}$ & $\begin{array}{c}\text { Typical Differential } \\
\text { Power at Output Con- } \\
\text { tact (dBm) }\end{array}$ & $\begin{array}{c}\text { Typical PA } \\
\text { Current } \\
\text { (mA) }\end{array}$ \\
\hline \hline 00 & -27.6 & 1.7 \\
\hline 04 & -20.6 & 2.5 \\
\hline 08 & -17.7 & 3.8 \\
\hline 0C & -16.3 & 6 \\
\hline 1C & -15.7 & 6.1 \\
\hline 2C & -15.2 & 6.1 \\
\hline 3C & -14.6 & 6.1 \\
\hline 4C - PWR 4 & -8.9 & 6.9 \\
\hline 5C & -8.2 & 7 \\
\hline 6C & -7.5 & 7.1 \\
\hline 7C & -7.1 & 7.2 \\
\hline 8C - PWR 8 & -1.6 & 9.3 \\
\hline 9C & -1.1 & 9.6 \\
\hline AC & -0.7 & 9.9 \\
\hline BC - PWR 11 & -0.3 & 10.2 \\
\hline CC & 1.3 & 12.2 \\
\hline DC & 1.9 & 13.6 \\
\hline EC & 2.5 & 16.3 \\
\hline FC & 2.6 & 16.6 \\
\hline FD & 3.2 & 16.8 \\
\hline FE & 3.7 & 16.9 \\
\hline FF - PWR 15 & 4.1 & \\
\hline
\end{tabular}

\section{Data Collection Experimental Setup}

In order to collect the data for all the experiments the receiving board was connected to a laptop through a serial port connection. All the incoming packets were thus logged. In order to get a broad range of results for analysis we varied both the packet size and transmit power level for all experiments. For this, we set "small" packets as 3 data bytes, "medium" packets as 60 data bytes, and "large" packets as 100 data bytes in size. A different delay function for each packet size was programmed in the transmitter between each packet transmission in order to not overfill the receive buffer. The structure of the packet contained the node ID as the first byte, the second byte was the sequence number, and the third byte contained the transmit power level. The remaining bytes for the larger packets were filled with ' $\mathrm{X}$ 's. Table 1 shows the various transmit power levels that can be achieved by the SARD boards. The transmit power is adjusted by calling a power adjust function in the program code and can be set per packet. Minimum power corresponds to setting the power adjust (PA) register to 00. Nominal power, which is the default for these 
nodes, sets the PA register value to $\mathrm{BC}$. To transmit at maximum power, the register value is set to FF. By choosing hexadecimal numbers in between these values, one can fine tune the transmit power.

The wireless radio is programmable to 16 selectable channels in the $2.4 \mathrm{GHz}$ ISM band where each channel occupies $3 \mathrm{MHz}$. Channels are centered at $5 \mathrm{MHz}$ from each other. This gives a $2 \mathrm{MHz}$ gap between each pair of channels. For all range experiments, the nodes were fixed to channel 5, which corresponds to $2.430 \mathrm{GHz}$. Channel 15, 2.480GHz, was used for the non-symmetric links experiment as well as all interference experiments.

\section{EXPERIMENTAL EVALUATION}

In the subsections below, we describe the details of the setups and the obtained results from three different sets of experimental scenarios: (i) transmission range, (ii) non-symmetric links, and (iii) three-node interference. Unless otherwise specified, in the discussions below we refer to the transmitter node as $N_{T x}$, receiver node as $N_{R x}$, and the interfering (jamming) node as $N_{\text {Int }}$.

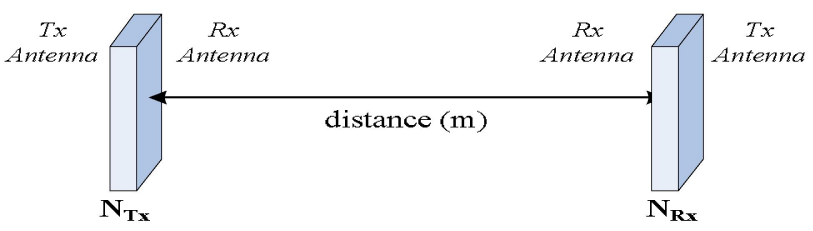

Figure 1. Range Test Experiment Setup

\section{A. Transmit Range}

The first set of experiments we consider is a simple range test. As shown in Figure 1, $N_{T x}$ and $N_{R X}$ are placed with receive antennas facing each other, while their transmit antennas are on opposite sides. We log the number of missed packets from each set, allowing us to form a basic idea of how far the nodes can transmit at the different power levels, heights, and environments. Thus, we varied the orientation and height of the boards as well as performing the experiments both indoor and outdoor.

In this case, $N_{T x}$ was programmed to send 256 packets at four different transmit power levels. In order to capture the results of transmitting at different power levels we chose the power levels $4,8,11$, and 15 . The typical power and current numbers for these power levels can be found in table 1. $N_{R x}$ was programmed to receive and $\log$ all packets using the connected PC.

We varied the orientation, height, packet size, and transmit power levels of the SARD devices. Figure 2 shows a SARD device with a flat (horizontal) antenna orientation. We studied the packet loss rate both indoors and outdoors with the flat antenna orientation, $1 \mathrm{~m}$ above the ground. We also studied packet loss percentages with the SARD devices at $2 \mathrm{~m}$ above the ground with both the vertical and standing antenna orientations as shown in figures Figure 3 and Figure 4 respectively. Finally, we ran experiments with the devices $15 \mathrm{~cm}$ above the ground with the antenna in the standing position.

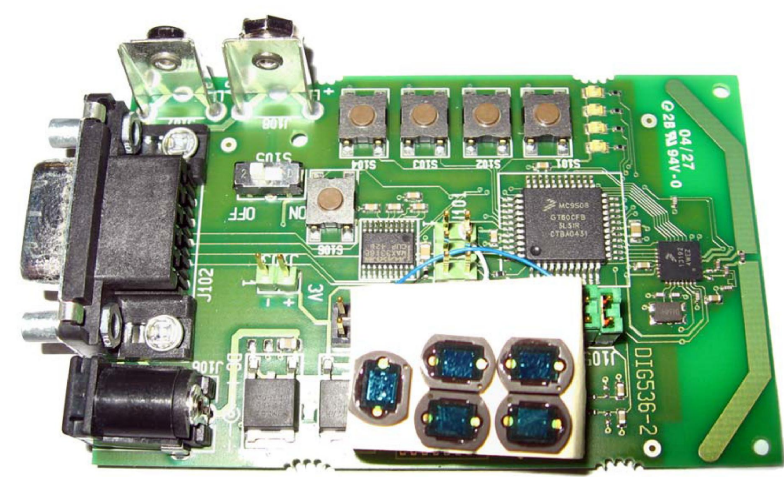

Figure 2. SARD board in "flat" antenna orientation.

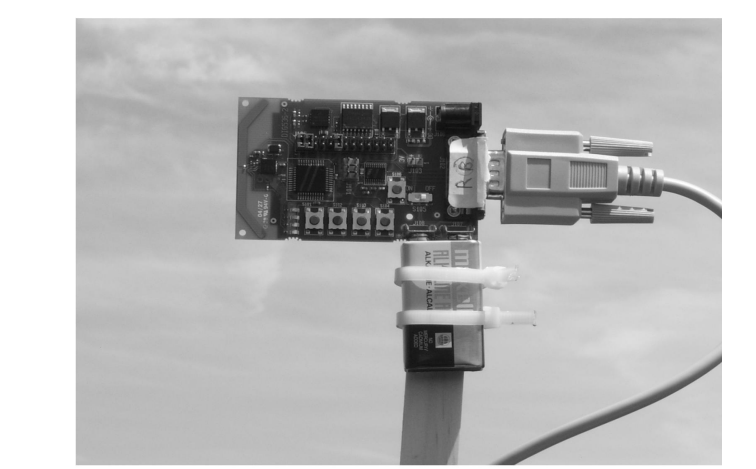

Figure 3. SARD board, $2 \mathrm{~m}$ high and "vertical" antenna.

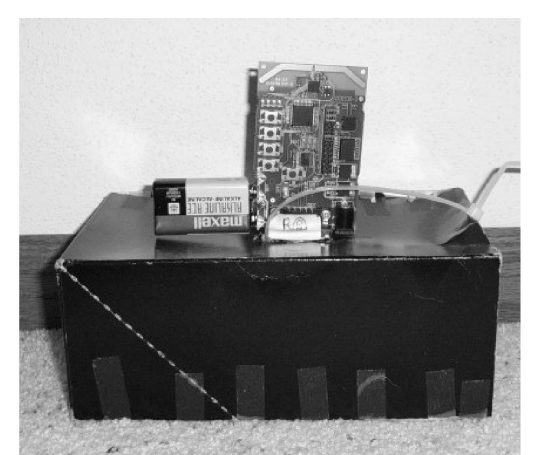

Figure 4. SARD board, $15 \mathrm{~cm}$ high and "standing" antenna.

\section{1) Indoor - Im height, flat antenna}

Figure 5 shows three graphs of our indoor range experiments in a corridor of the UW Madison Engineering Hall that is $61 \mathrm{~m}$ long and $2 \mathrm{~m}$ wide. As expected, and as the figures show, indoor transmission ranges can be quite unpredictable. The figures show that their exists 
larger packet losses around $20 \mathrm{~m}$ and $55 \mathrm{~m}$ while other distances stay under $2 \%$ packet loss. These abnormal results could be due to interference from other wireless devices or destructive multi-path effects.

\section{2) Outdoor - $1 \mathrm{~m}$ height, flat antenna}

Figure 6 details the results of experiments done with both nodes placed on stools $1 \mathrm{~m}$ above the ground outdoors, in a large open field approximately 600×600 meters. The figures show that at all power levels packet losses begin to occur at approximately $120 \mathrm{~m}$. At power 4 , packet loss reaches $100 \%$ around $150 \mathrm{~m}$. Furthermore, at $305 \mathrm{~m}$, and all power levels, a large majority of the packets are lost. Finally, the results show that on average, the packet loss percentage seems to increase as the size of the transmitted packet increases.

\section{3) Outdoor $-2 m$ height, standing antenna}

Figure 7 shows the results of experiments, in the same outdoor environment as above, with both nodes mounted $2 \mathrm{~m}$ above the ground on wooden stakes. These particular results show that outdoor transmission can be unpredictable as well. At around $60 \mathrm{~m}$, packet loss percentages are very high for all power levels. This could

be due to other wireless interference or the orientation of the antenna. At this height and antenna orientation, the results show a gradual packet loss increase for power 4 . All other power levels stay below $25 \%$ packet loss except for the abnormal $60 \mathrm{~m}$ case.

\section{4) Outdoor - $2 m$ height, vertical antenna}

Figure 8 shows the results with the nodes mounted, again 2 meters off the ground on stakes as in the previous case. However, for these experiments we changed the antenna orientation of the transmitter and receiver to a vertical position shown in Figure 3. Again, we observed the $60 \mathrm{~m}$ anomaly. However, at all power levels above 4 , the packet loss stays below $10 \%$ even at our farthest test distance of $305 \mathrm{~m}$.

\section{5) Outdoor $-15 \mathrm{~cm}$ height, standing antenna}

Figure 9 shows the results of range experiments done in the same outdoor environment as above, with the nodes placed $15 \mathrm{~cm}$ above the ground in the standing position as depicted in Figure 4. As can be seen from the figures, packet loss percentages increase much more rapidly with distance due to the ground effect limiting transmission ranges. At power 4 , the nodes experience $100 \%$ packet loss at $24 \mathrm{~m}$ for all packet sizes. Furthermore, most packets are lost at $37 \mathrm{~m}$ for 60 byte and 100 byte packets at all power levels. Also, these figures again show the trend that the larger the packet size the greater the percentage of packet loss.
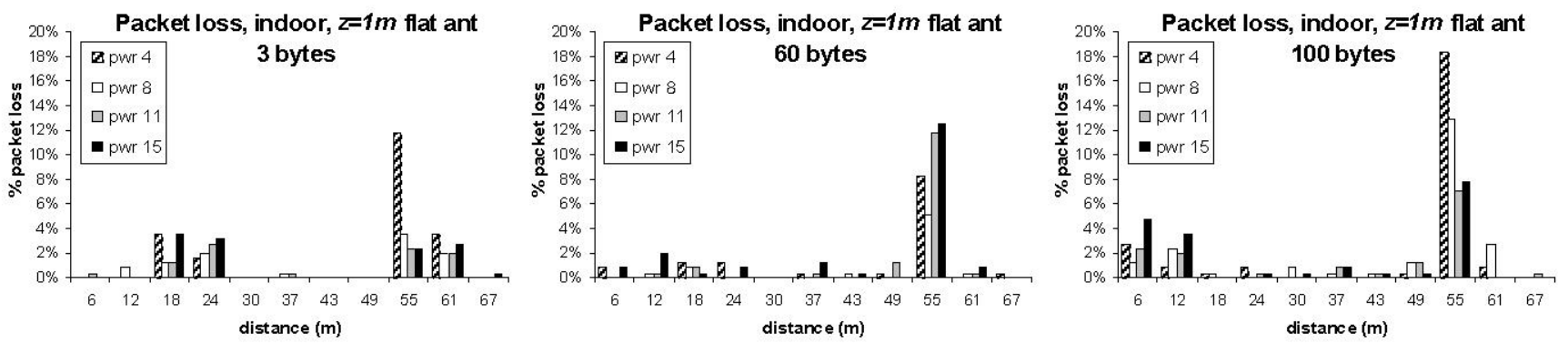

Figure 5. Indoor range test, $1 \mathrm{~m}$ node height, flat antenna orientation, three packet sizes.
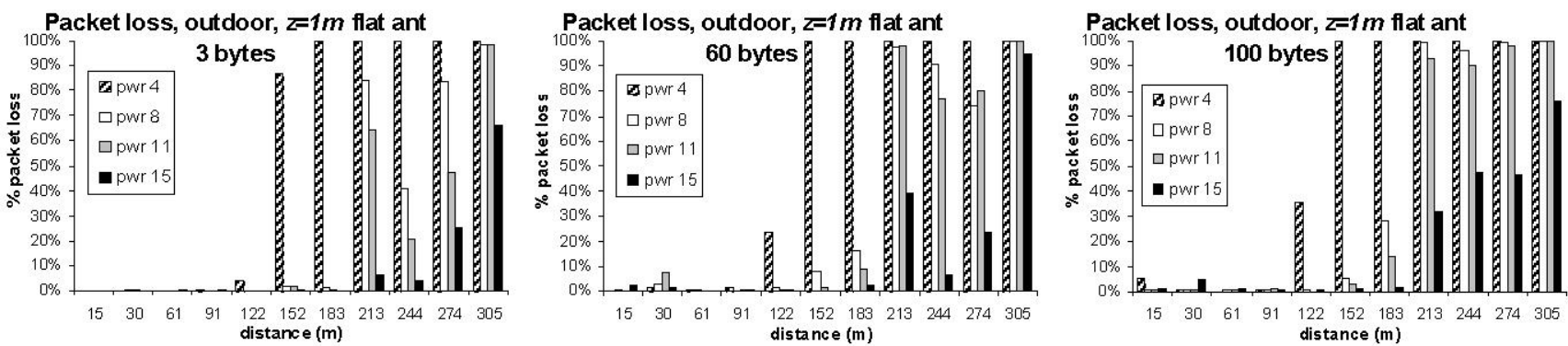

Figure 6. Outdoor range test, $1 \mathrm{~m}$ node height, flat antenna orientation, three packet sizes. 

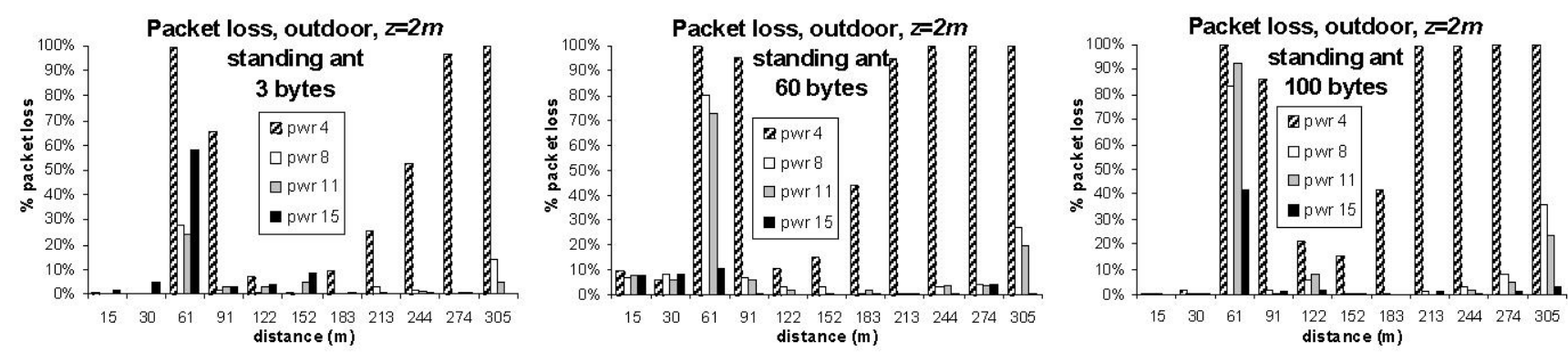

Figure 7. Outdoor range test, $2 \mathrm{~m}$ node height, standing antenna orientation, three packet sizes.
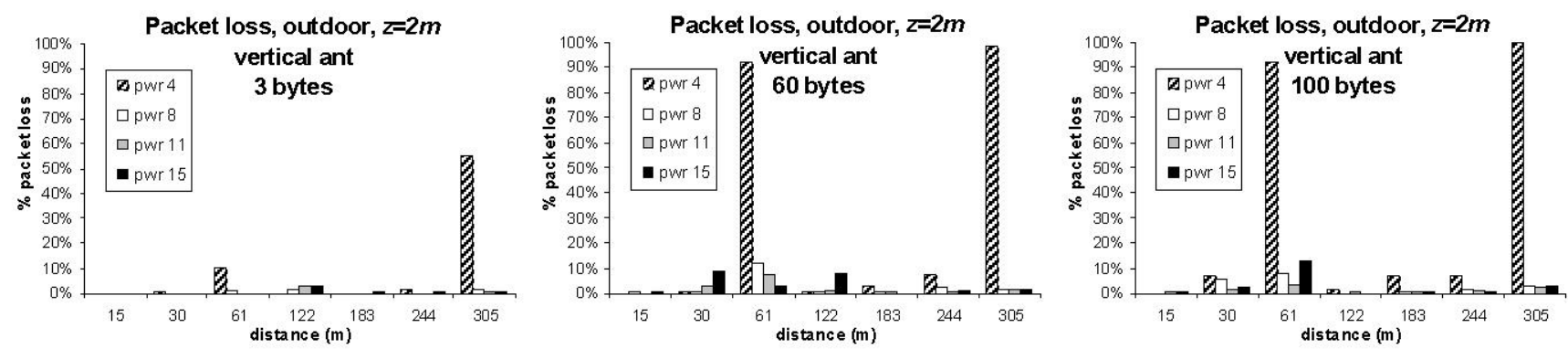

Figure 8. Outdoor range test, $2 \mathrm{~m}$ node height, vertical antenna orientation, three packet sizes.
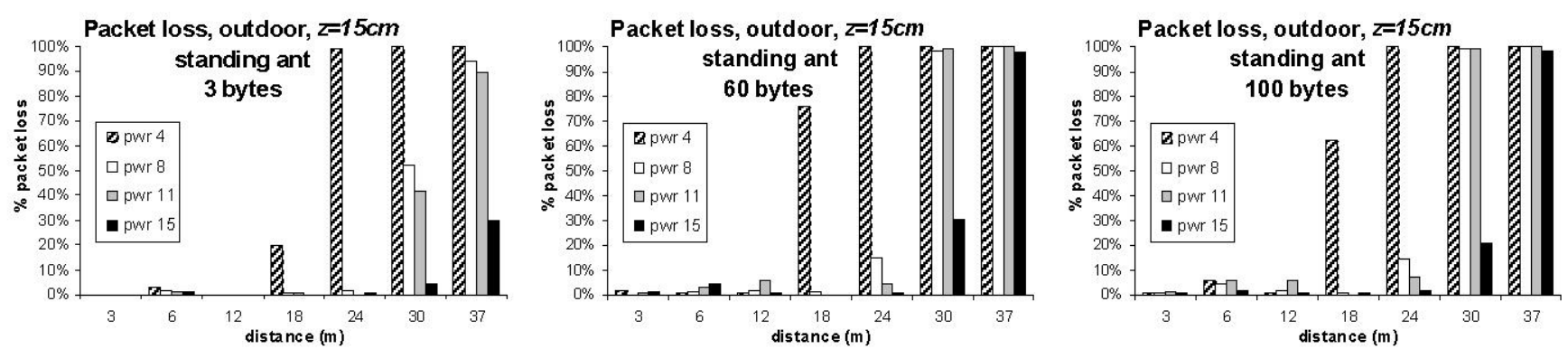

Figure 9. Outdoor range test, $15 \mathrm{~cm}$ node height, standing antenna orientation, three packet sizes.

As expected, from analysis of these figures one can verify the role that node height plays on packet transmission ranges. However, at the same time, antenna orientations also directly affect the packet loss rates of these devices. Furthermore, there are a number of factors such as external interference that could also adversely affect the transmission between these devices that could produce unpredictable results, which also appeared in our range figures. Finally, on average, packet loss was found to increase with the increase in packet size. This trend seems to hold true due to the simple fact that larger packets tend to have a higher packet error rate than smaller packets.

\section{B. Non-Symmetric Links Experiment}

The SARD board contains a transmit and a receive dipole antennae printed on opposite sides of the board. Our non-symmetric links experiment is intended to study the impacts of this non symmetry in the antennae positions. Figure 10 shows the setup of this experiment and the orientations of the boards relative to each other. This setup differs from the range experiments in the previous subsection in that the transmit antenna on each nodes faces the same absolute direction. Thus, the transmit antenna of node A faces the receive antenna of node $B$, while the transmit antenna of node $B$ and the receive antenna of node A face away from each other. The nodes were placed $15 \mathrm{~cm}$ above the ground, indoors, with the antenna in the standing position.

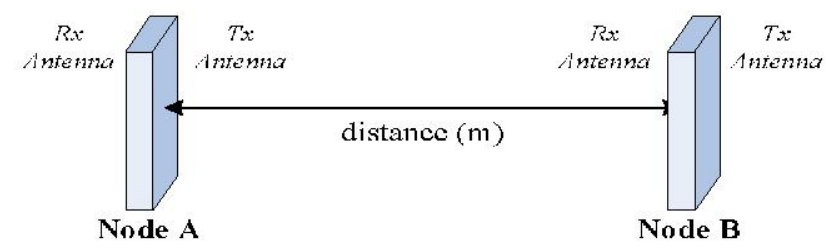

Figure 10. Experimental setup for non-symmetric links. 

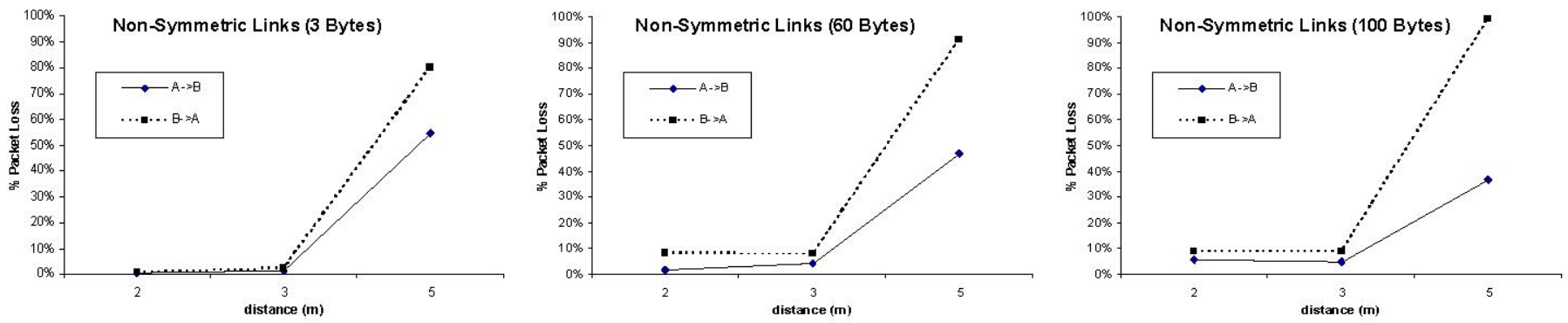

Figure 11. Effect of the non-symmetric links due to antenna placements on packet transmission rates.

In this case, each node was programmed to be both a transmitter and a receiver. The transmit power was set to minimum power, which corresponds to the register value of 00 (hex). Each node sent 1024 packets to the other while the receiving node recorded the number of packets missed from the entire transmission.

Figure 11 shows the results of this experiment. Analyzing these figures we see that the transmission from node $\mathrm{A}$ to node $\mathrm{B}$ produces a greater packet loss percentage than the transmission from node $\mathrm{B}$ to node $\mathrm{A}$. These results confirm our intuition that the differing placement of the transmit and receive antennae does in fact create a non-symmetric communication link between the two nodes which is a contributor to the directionality of the transmission and interference patterns that we observe later on. Again, here as the packet sizes and the distance between nodes $\mathrm{A}$ and $\mathrm{B}$ increase, so do the packet loss percentages.

In addition to the above, this experiment also illustrates the very limited range of successful transmission at minimum power with this particular hardware. Results from the 60-byte and 100-byte packets in Figure 11 show that transmitting from $\mathrm{B}$ to A with larger packets sizes results in nearly $100 \%$ packet loss at a distance of only $5 \mathrm{~m}$. This further shows how the packet loss can be directly affected by the transmit power setting.

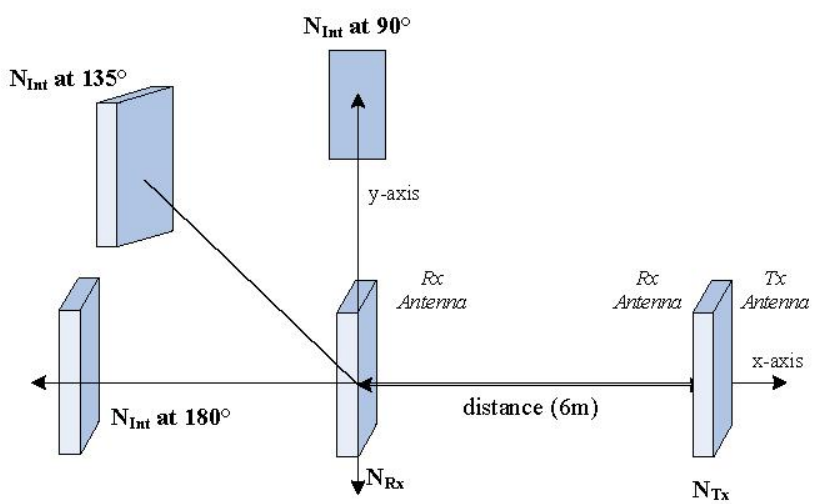

Figure 12. Experimental setup changing positions of $N_{I n t}$.

\section{Three Node Interference Experiment}

\section{1) Changing Position of $N_{\text {Int }}$}

For the following set of experiments, a third interferer node $N_{\text {Int }}$ was introduced to see how 802.15.4 interference affects a the transmission between the $\mathrm{N}_{T x}$ and $\mathrm{N}_{\mathrm{Rx}}$. Figure 12 illustrates the setup for this set of experiments. Nodes $N_{T x}$ and $N_{R x}$ are placed at a constant distance of $6 m$ apart facing each other. All nodes were placed $15 \mathrm{~cm}$ above ground, outdoors, with their antenna in the standing position as in Figure 4. We changed the position of the interferer with respect to the active transmit/receive link. The interferer $N_{\text {Int }}$ was placed at several distances along the $+\mathrm{y}$ axis $\left(90^{\circ}\right.$ line $), 135^{\circ}$ line, and $-\mathrm{x}$ axis $\left(180^{\circ}\right.$ line). The interferer's orientation was kept constant, with its receive antenna pointed towards $N_{R x}$ to minimize the number of changing parameters.

The power level of $N_{T x}$ and $N_{I n t}$ are both set at $4 . N_{I x}$ sent 1024 packets to $N_{R x}$, while $N_{I n t}$ continuously sent 100 byte jammer packets with no delay. The packet transmission was logged at the $N_{R x}$ via the serial port connection on the SARD device.

Figure 14 shows the results of changing the position of the interferer as well as altering the distance of $N_{\text {Int }}$ from $N_{R x}$. The distances of $N_{I n t}$ were carefully chosen such that $N_{R x}$ and $N_{T x}$ would not be able to successfully receive (hear) the packets from $N_{\text {Int }}$. For all packet sizes, when the interferer is $90^{\circ}$ with respect to the $\mathrm{Tx} / \mathrm{Rx}$ link, $0 \%$ packet loss was found to be at approximately a distance of $11 \mathrm{~m}$. When the interferer was at both $135^{\circ}$ and $180^{\circ}$ lines, $0 \%$ packet loss occurred around $20 \mathrm{~m}$ for all packet sizes. If the $135^{\circ}$ and $180^{\circ}$ result lines were extrapolated, the packet loss percentages at lower distances would be significantly larger than that of the $90^{\circ}$ results. These results clearly illustrate that in this case, nodes placed $90^{\circ}$ with respect to the $\mathrm{Tx} / \mathrm{Rx}$ link produce much less interference than those at the other angles at the given orientations. 


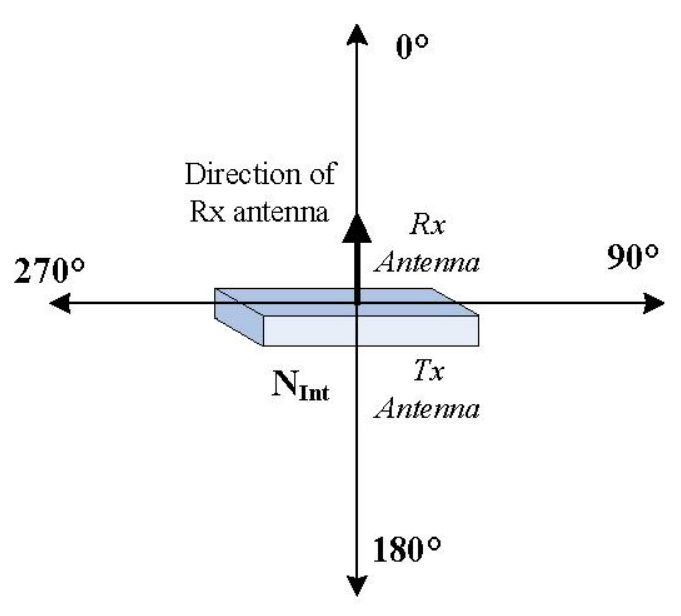

Figure 13. Orientations of the interferer $N_{i n t}$.

\section{2) Changing Orientation of Interferer $N_{\text {Int }}$}

In these set of experiments, we focus on the effects of changing the orientation of $N_{\text {Int }}$ on the ongoing transmissions. The setup of the $\mathrm{Rx} / \mathrm{Tx}$ nodes is the same as the last set of experiments, detailed in Figure 12. Interferer $N_{\text {Int }}$ was placed on both the $+\mathrm{y}$ axis $\left(90^{\circ}\right)$ and $-\mathrm{x}$ axis $\left(180^{\circ}\right)$ at different antenna orientations. These orientations are shown in Figure 13. Again, all nodes were placed $15 \mathrm{~cm}$ above the ground, outdoors, with their antenna in the standing position. This set of results illustrates that not only the position of the interferer, but also the orientation, affect a $\mathrm{Tx} / \mathrm{Rx}$ transmission.

Here, the transmitter $N_{T x}$ and receiver $N_{R x}$ were positioned $6 \mathrm{~m}$ apart, as depicted in Figure 12. $N_{\text {Int }}$ was placed in three different angles positions $+y$ axis $\left(90^{\circ}\right)$, $135^{\circ}$, and $-\mathrm{x}$ axis $\left(180^{\circ}\right)$. The power level of $N_{T x}$ and $N_{I n t}$ are both set at 4 . $N_{T x}$ sent 1024 packets to $N_{R x}$, while $N_{I n t}$ continuously sent 100 byte jammer packets with no delay. The packet transmission was logged at the $N_{R x}$ via the serial port connection on the SARD device. Figure 15 and Figure 16 show the results of changing the orientation of the interferer while also changing its distance $N_{R x}$. Distances for these sets of experiments were chosen to see just how much interference $N_{\text {Int }}$ can have even if $N_{R x}$ can not decode the interference packets. The orientations of $N_{\text {Int }}$ are outlined in Figure 13.

Figure 15 shows the interference results while changing $N_{\text {Int }}$ 's orientation along the $+\mathrm{y}$-axis. These figures
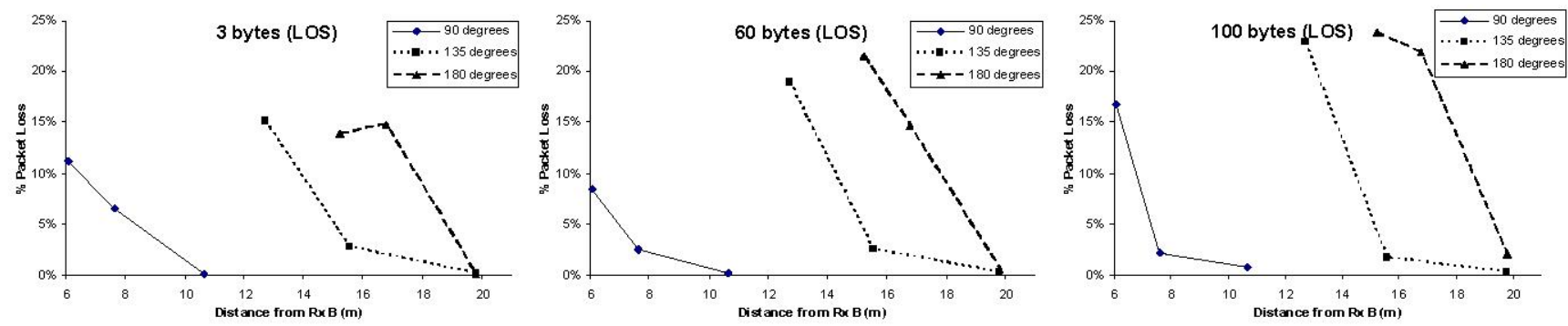

Figure 14. Effects of the position of the interfering node on throughput while always facing the receiver.
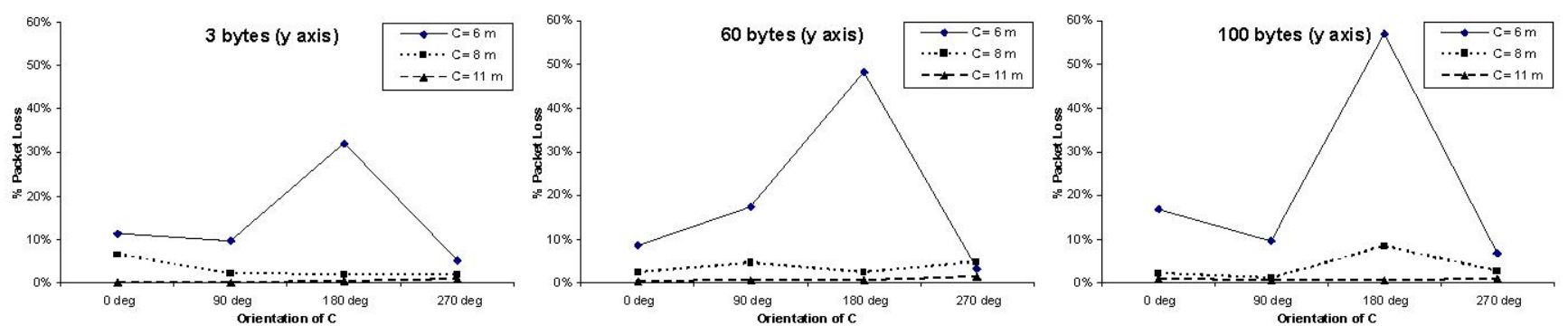

Figure 15. Effects of the positions and orientation of the interfering node on throughput at positions along the $v$ axis.
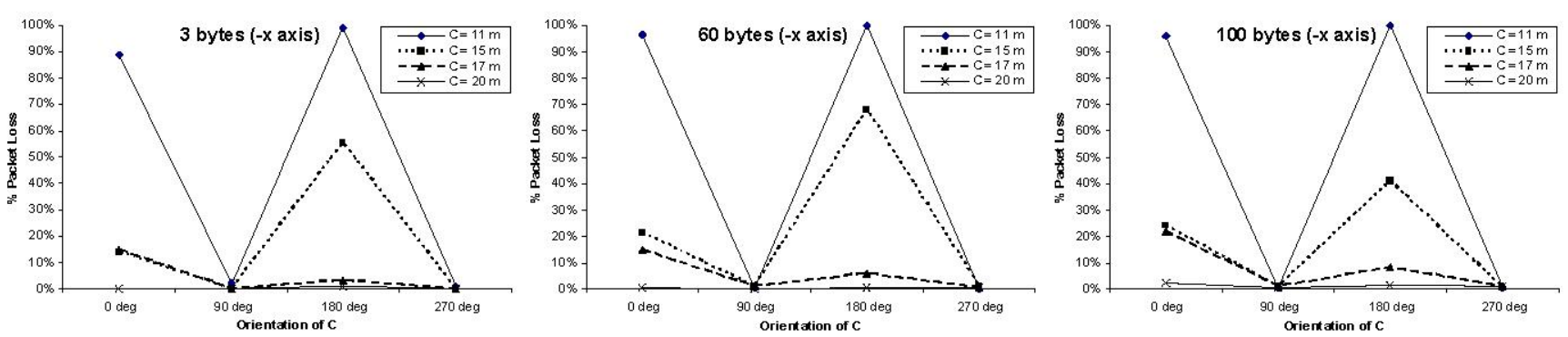

Figure 16. Effects of the orientation of the interfering node on throughput at positions along the $-\mathrm{x}$ axis. 
show that when $N_{\text {Int }}$ is only $6 \mathrm{~m}$ away from $N_{R x}$, and its antenna orientation is at $180^{\circ}$, packet loss is more prevalent than in any other scenario. It can also be seen from the results in this figure that at $11 \mathrm{~m}$, for all interferer orientations, packet loss was found to be very minimal.

Figure 16 shows the interference results while changing $N_{\text {Int }}$ 's orientation along the -x-axis. These figures show that for all packet sizes, when the interferer is $11 \mathrm{~m}$ away, nearly $100 \%$ packet loss occurs for orientations $0^{\circ}$ and $180^{\circ}$. At this distance and orientations of $N_{\text {Int }}$, the $N_{R x}$ was getting completely jammed by the interferer. Also, around $40 \%$ to $50 \%$ packet loss was experienced at $15 \mathrm{~m}$ when $N_{\text {Int }}$ was oriented at $180^{\circ}$. This again shows that the antenna is not perfectly omnidirectional and the Tx antenna is printed on the back of the SARD device. Packet loss percentages were found to be minimal at all distances when the $N_{\text {Int }}$ was oriented at $90^{\circ}$ and $270^{\circ}$ with respect to the receiver. Finally, with the interferer at $20 \mathrm{~m}$ with respect to all interferer orientations, packet loss percentages were negligible.

\section{3) Analysis of three-node interference results}

These interference experiments show that not only the position and distance of the interferer, but the orientation also has a differing affect on the $\mathrm{Tx} / \mathrm{Rx}$ transmission. Thus, care must be taken to consider antenna orientations, node positions, and distances between nodes in order to maximize the throughput of the network.

\section{SCHEDULING FOR INTERFERENCE MINIMIZATION USING INTERFERENCE WEIGHTED EDGE COLORING}

Using the insights learned from the experiments above, in this section, we briefly address the problem of scheduling the communications between different pairs of nodes (radios) to the same or different periodic timeslots in such a way that interference is minimized and, therefore, the effective bandwidth of the network is maximized. We start by presenting an informal and graph theoretic formulation of the problem and explaining our rationale for its abstraction. After proving that the problem is NP-complete, we introduce a new recursive algorithm for solving it. We conclude by proposing preprocessing steps for further enhancement of the performances of the new approach.

We model wireless ad-hoc multi-hop network as a weighted graph $G(V, E, \Phi)$. Each vertex $v_{i} \in V$ corresponds to a radio in the network. Each directed edge $e_{i j} \in E$ indicates a communication link between the corresponding radios represented by the vertices $v_{i}$ and $v_{j}$. Each edge has an associated weight $w_{i j}$ that corresponds to successful packet reception rate of the link (e.g. cor- responding to the data obtained in the previous sections). Finally, for each node triple $\left\{v_{a}, v_{i}, v_{j}\right\}$ consisting of a node $v_{a}$ and an edge $e_{i j}$ we have a weight $\varphi_{i} \in \Phi$ that indicates the reduction in the reception rate of $e_{i j}$ if node $v_{a}$ is simultaneously active. This is motivated by our observations above that an interfering node will interfere differently with respect to each communication edge of a receiver.

The goal is to determine the number of time slots $T$ and an assignment of edges to time slots such that the packet loss due to interference is minimized. We assume that the interference influence from different nodes is additive. This assumption can be easily altered with very minimal changes in the optimization algorithm to otherwise combined interference functions. Note that we have to simultaneously minimize both the number of time slots $T$ and the loss in bandwidth due to interference. We address this problem by conducting linear search along the number of slots $T$.

Hence, our problem can be defined using the following graph-theoretic formulations:

Problem: Weighted Edge Coloring

Instance: Given is a weighted graph $G(V, E, \Phi)$ where interference weight $\Phi$ are associated with nodes and communication edges they interfere with, an integer $T$, and a real number $C$.

Question: Is there a coloring of $E$, i.e., a partition of $E$ into disjoint sets $E_{1}, \ldots, E_{T}$, such that the sum of weights over all sets between each of the edges in the same set and any node that is incident to any other edge in the same set is less than $C$ ?

We prove that the problem is NP-complete by observing that it reduces to minimum edge coloring [9] by assigning unit weight $\varphi_{i}=1$ to any triple consisting of an edge and a node that is the neighbor of one node incident on the edge.

Our algorithm here is a generalization of the Leighton recursive algorithm for edge coloring [16]. The algorithm at each step iteratively forms an independent set that has the largest number of edges toward still nonaddressed nodes. The set is formed using a greedy heuristic. In addition of addressing a somewhat different problem, edge coloring instead of node coloring, and using a different objective function, the amount of interference of the nodes in the selected set and the rest of still uncolored graph, we introduce three algorithmic novelties to improve the performance:

First we form several $(\mathrm{k}=3)$ independent sets simultaneously. Second, each set is formed using probabilistic iterative improvement with large number of restarts 
(usually several hundred). Finally, when the number of nodes is small, both when the sets are formed and overall, we apply an optimal branch-and-bound algorithm.

There are several important observations that can be used to make the algorithm more effective when applied to the targeted task of scheduling for interference minimization. The first is that we can consider traffic between the nodes and penalize proportionally more for loss of bandwidth on more heavily used links/edges. Furthermore, we can consider only edges of high quality (i.e., high reception rate of with high ratio of the reception rate and the distance between the two corresponding nodes) and remove all other edges as preprocessing nodes because other edges anyhow will not be used.

A detailed treatment of the algorithmic details involved in the above is beyond the scope of this paper. For a number of related research efforts, please refer to references [17] to [21]. Furthermore, we are currently working to experimentally evaluate the performance of this algorithm since a small number of nodes is not sufficient to obtain any meaningful results. In the near future, we are aiming to carry out detailed experiments with a 27 node hardware test bed.

\section{CONCLUSION}

In this paper we presented an in-depth experimental study of the packet transmission success rates that can be realized using links formed with a commercially available IEEE 802.15.4 radio transceiver. We began our discussion by a basic study of the transmission ranges under a variety of conditions and at the various available transmit power levels. We then shifted our focus to study the non-symmetric links that result from the transmit and receive antennae that are printed on different sides of the hardware board. This non symmetry, combined with other packaging factors and physical constraints create RF radiation patterns that result in interferences that change significantly with the relative positions and the orientations of the nodes. The detailed experimental results with respect to changing the parameters were followed by a discussion on how this data and observations can be used to create an interference model that more accurately captures the underlying directionalities as opposed to more simplistic models that depend purely on distance between nodes. The discussion of the interference model that essentially corresponds to a graph with node-to-link edge interference weight was augmented with a discussion on an algorithm that can be used to schedule nodes such that interference is minimized.

\section{REFERENCES}

[1] G. Anastasi, et. al., "Performance Measurements of Motes Sensor Networks." Proceedings of the 7th ACM international symposium on Modeling, analysis and simulation of wireless and mobile systems, 2004.

[2] A. Woo, D. Culler, "A transmission control scheme for media access in sensor networks." ACM Mobicom 2004.

[3] G. Zhou, T. He, J. Stankovic, T. Abdelzaher, "RID: Radio Interference Detection in wireless sensor networks." IEEE Infocom 2005.

[4] J. Zhao and R. Govindan, "Understanding packet delivery performance in dense wireless sensor networks." ACM Sensys 2003.

[5] N. Reijers, G. Halkes, and K. Langendoen, "Link Layer measurements in Sensor Networks." MASS 2004.

[6] M. Petrova, J. Riihijarvi, P. Mahonen, and S. Labella, "Performance Study of IEEE 802.15.4 Using Measurements and Simulations." WCNC 2006.

[7] J. Padhye, S. Agarwal, V. Padmanabhan, L. Qiu, A. Rao, B. Zill, "Estimation of Link Interference in Static Multi-hop Wireless Networks." IMC 2005 .

[8] D. Aguago, J. Bicket, S. Biswas, G. Judd, and R. Morris, "Link-level measurements from an 802.11b mesh network." SIGCOMM, 2004.

[9] Freescale Semiconductor, SARD User's Guide, 1.5 ed., August 2005. Document number MC13192SARDUG.

[10] Freescale Semicondutor MC9S08GB /GT Data Sheet, 2.3 ed., December 2004. Document number MC9S08GB60.

[11] Freescale Semiconductor, MC13192/MC13193 Data Sheet, 1.4 ed., May 2006. Document number MC13192RM.

[12] Freescale Semiconductor, MC13192/MC13193, 2.9 ed., August 2005. Document number: MC13192.

[13] D. Lymberopoulos, Q. Lindsey, and A. Savvides, "An Empirical Characterization of Radio Signal Strength Variability in 3-D IEEE 802.15.4 Networks Using Monopole Antennas." Proceedings of the Second European Workshop on Sensor Networks (EWSN 2006).

[14] Freescale Semiconductor, Simple Media Access Controller (SMAC) Users Guide, 1.2 ed., April 2005. Document number SMACRM.

[15] M. R. Garey and D. S. Johnson. Computers and Intractability: A Guide to the Theory of NP-Completeness. W.H. Freeman and Company, 1979.

[16] F. T. Leighton, "A graph coloring algorithm for large scheduling problems." Journal of Research of the National Bureau of Standards, 84:489, 1979.

[17] Kamal Jain, Jitendra Padhye, Venkata N. Padmanabhan and Lili Qiu, "Impact of Interference on Multi-Hop Wireless Network Performance." Wireless Networks, Volume 11, Number 4, pp. 471 -487, July 2005.

[18] Wieselthier, J.E. Barnhart, C.M. Ephremides, A. "A neural network approach to routing without interference in multihop radio networks." IEEE Trans. on Communications Vol: 42:1, pp. 166-177, Jan 1994.

[19] A.A. Bertossi, M.A. Bonuccelli, "Code assignment for hidden terminal interference avoidance in multihop packet radio networks." IEEE/ACM Trans. Networking, vol. 3, no. 4, pp. 441-449, August 1995.

[20] R. Nelson, L. Kleinrock, "Spatial TDMA, A collision-free Multihop Channel Access Protoco1." Mobile Computing and Communications Review, Volume 1, Number 2, IEEE Transactions on Communications, Vol. COM-33, No. 9, September 1985.

[21] I. Cidon and M. Sidi, "Distributed Assignment Algorithms for Multihop Packet Radio Networks." IEEE Transactions on Computers, vol. 38, No. 10, pp. 1353-1361, Oct. 1989.

[22] T. Sun, et. al., "Measuring Effective Capacity of IEEE 802.15.4 Beaconless Mode." IEEE WCNC 2006.

[23] Shan Lin, et. al., "ATPC: Adaptive Transmission Power Control for Wireless Sensor Networks", ACM SenSys, Nov. 2006. 